\title{
Method for in vitro differentiation of bone marrow mesenchymal stem cells into endothelial progenitor cells and vascular endothelial cells
}

\author{
QIHONG WANG ${ }^{1}$, WEIFENG ZHANG ${ }^{1}$, GUIFEN HE ${ }^{2}$, HUIFANG SHA $^{3}$ and ZHE QUAN ${ }^{4}$ \\ ${ }^{1}$ Department of Neurosurgery, Ruijin Hospital, Shanghai Jiaotong University School of Medicine, Shanghai 200025; \\ ${ }^{2}$ School of Life Science, Fudan University, Shanghai 200433; ${ }^{3}$ Chest Tumor Research Institute of Shanghai Chest Hospital, \\ Shanghai Jiaotong University, Shanghai 200030; ${ }^{4}$ Department of Neurosurgery, Fengxian District Central Hospital \\ (Branch Hospital of Shanghai Sixth People's Hospital), Shanghai Jiaotong University, Shanghai 201400, P.R. China
}

Received June 6, 2016; Accepted November 10, 2016

DOI: $10.3892 / \mathrm{mmr} .2016 .5953$

\begin{abstract}
Vascular development is a regulated process and is dependent on the participation and differentiation of many cell types including the proliferation and migration of vascular endothelial cells and differentiation of endothelial progenitor cells (EPCs) to mesodermal precursor cells. Thus, reconstitution of this process in vitro necessitates providing ambient conditions for generating and culturing EPCs in vitro and differentiating them to vascular endothelial cells. In the present study, we developed methods to differentiate bone marrow mesenchymal stem cells (MSC) into EPCs and to vascular endothelial cells. Bone marrow MSC from canines and human sources were differentiated in vitro in to EPCs. These EPCs were able to express a variety of endothelial markers following 7 days in culture. Further culturing led to the appearance of an increased number and proportion of endothelial cells. These cells were stable even after 30 generations in culture. There was a gradual loss of CD31 and increased expression of factor VIII, VEGFR and CD133. VEGF being highly angiogenic, helps in the vascular development. These results provide the basis for the possible development of vasculature in vitro conditions for biomedical applications and in vivo for organ/tissue reconstruction therapies.
\end{abstract}

\section{Introduction}

Vasculature in the body occurs by the arrangement of different types of cells throughout the development process

Correspondence to: Dr Zhe Quan, Department of Neurosurgery, Fengxian District Central Hospital (Branch Hospital of Shanghai Sixth People's Hospital), Shanghai Jiaotong University, 6600 Nanfeng Road, Shanghai 201400, P.R. China

E-mail: zhe_quan_1@163.com

Key words: bone marrow mesenchymal stem cells, endothelial progenitor cells, differentiation, CD31, CD133, vascular endothelial growth factor, vasculature and later during growth into adulthood. Endothelial cells form the luminal side of a blood vessel while pericytes or mural cells contribute to the formation of the outer surface. As the vessel size increases, smooth muscle cells and elastic and collagen fibers along with fibroblasts take part in structural organization (1). Blood vessel formation can be of two types, angiogenesis and vasculogenesis. Angiogenesis is a process of proliferation and sprouting of pre-formed blood vessels, whereas vasculogenesis is due to the de novo genesis of vessels from circulating progenitor cells and stem cells, mostly derived from bone marrow, in the blood (2). Angiogenesis is of particular significance in tumor growth and progression while vasculogenesis is important for the recovery of cardiac muscle following ischemia, as it can help restore heart muscle function after heart failure (3). There are different types of adult stem cells in the bone marrow types of stem cells, and these include hematopoietic stem cells (HSC) or hematopoietic progenitor cells (HPC), which are $\mathrm{CD} 34^{+}$cells and mesenchymal stem cells (MSC). In various animal models of ischemia, these stem cells have been shown to be effective in improving vessel formation and in restoring heart muscle function in vivo (4). MSC from bone marrow can differentiate into endothelial progenitor cells (EPCs), which are known to express hematopoietic markers, CD34 or CD133 and also vascular endothelial growth factor (VEGF) receptor 2.

Although different types of stem cells have been shown to have the potential to contribute to the generation of vasculature, disease conditions are likely to affect the ability and also availability of the stem cells and progenitor cells (5). Thus, factors that lead to diabetes and/or cardiovascular complications have been found to reduce the vascularization ability of the progenitor cells (6). Several advances have been made in stem cell therapy and considering that patient-derived stem cells may not have the necessary potential to differentiate to form vasculature, there is a need to develop methods for generating large quantity of the EPCs so that they can be used for therapeutic purposes.

In the present study, we developed methods to differentiate human and canine bone marrow MSC in to EPCs and to vascular endothelial cells, which were stable for extended 
period (30 generations) in culture and could form vessel-like structures in vitro and thus have the potential to be used in vivo and for therapeutic purposes.

\section{Materials and methods}

Ficoll stock solution was purchased from Amersham Pharmacia Biotech (Piscataway, NJ, USA); heparin was purchased from the Shanghai Biochemical Pharmaceutical Factory (Shanghai, China); trypsin was purchased from Sino-American Biotechnology Co., Ltd. (Shanghai, China); Dulbecco's modified Eagle's medium (DMEM) was purchased from Gibco (Grand Island, NY, USA); and vVEGF, $\beta$-FGF were purchased from R\&D Systems, Inc. (Minneapolis, MN, USA). ECGF was prepared by our research laboratory. Sources of antibodies were as follows: Sheep anti-rabbit monoclonal rhodamine antibody (dilution 1:5,000, Promega, Madison, USA; cat no.: AS1270); FITC-labeled mouse monoclonal CD133 antibody (1:50; Cell Signaling Technology, Danvers, MA, USA; cat no.: 60577), mouse monoclonal CD31 antibody (1:50; Cell Signaling Technology, Danvers, MA, USA; cat no.: 3568); mouse monoclonal CD34 antibody (1:50; Cell Signaling Technology, Danvers, MA, USA; cat no.: 79253S); rabbit polyclonal VEGF antibody (dilution: 1:200, Abcam, Cambridge, MA, USA; cat no.: ab2349); and goat polyclonal factor VIII antibody (dilution: 1:200, Abcam, Cambridge, MA, USA; cat no.: ab139391); and CD34 magnetic separation kit (Miltenyi Biotec, Bergisch Gladbach, Germany). All the procedures involving human bone marrow collection and preparation of bone marrow cells and animal tissues were approved by the Ethics Committee of Shanghai Jiaotong University School of Medicine. Signed written informed consent was obtained from the participants before the study. The study was also approved by the Animal Ethics Committee of Shanghai Jiaotong University Animal Center.

Isolation, culture, purification and passage of MSCs. Bone marrows were obtained from 6 patients (4 males and 2 females; 45-72 years) who were free of any types of cancer or bone metastases, admitted for thoracic surgery at the Shanghai Chest Hospital. Bone marrows were also obtained from 5 mongrel dogs of both genders under surgery after pentobarbital anesthesia. The bone marrow cavity was washed with $50 \mathrm{ml}$ phosphate-buffered saline (PBS), containing $50 \mu \mathrm{g} / \mathrm{ml}$ heparin, to collect bone marrow cell suspension.

Primary culture of MSCs. Gelatin-coated culture bottles were added with $8 \mathrm{ml}$ of DMEM containing $20 \%$ fetal bovine serum, $10 \mathrm{ng} / \mathrm{ml}$ ECGF, $10 \mathrm{ng} / \mathrm{ml}$ VEGF, $10 \mu \mathrm{ml}$ heparin, $1 \mathrm{ng} / \mathrm{ml} \mathrm{bFGF}$ and other cell growth factors at $37^{\circ} \mathrm{C}$ and inoculated with bone marrow cell preparation, and incubated in $5 \% \mathrm{CO}_{2}, 95 \% \mathrm{O}_{2}$ atmosphere at $37^{\circ} \mathrm{C}$. Cell attachment was monitored every day and the culture medium was changed after 2 days. The culture dishes were agitated gently to detach the red blood cells and other cells that did not adhere and removed these cells by washing in PBS 2-3 times. Several multiple clones of cells were formed and were continued to culture under the same conditions with fresh culture medium. Primary cells showed $\sim 80 \%$ confluency after 4-7 days, and were subcultured.
Isolation of MSCs and phenotypic characterization by flow cytometry. The cultured primary cells from bone marrow isolates were harvested after 3rd-4th generation, by washing with PBS once, followed by RPMI-1640 + $0.02 \mathrm{U}$ collagenase $+100 \mathrm{U} / \mathrm{ml}$ DNAse for $45 \mathrm{~min}$. The resulting cell suspension was filtered through $30-\mu \mathrm{m}$ filter, washed with PBS, and centrifuged at $1,500 \mathrm{x}$ g for $10 \mathrm{~min}$. The pelleted cells were suspended in $5 \mathrm{ml}$ PBS and mixed with $5 \mathrm{ml} \mathrm{Ficoll,}$ and centrifuged at $200 \mathrm{x}$ g for $25 \mathrm{~min}$, to separate karyocytes, which were further washed with PBS + EDTA twice, and counted. The cells were further processed for the separation and quantification of $\mathrm{CD} 34^{+}$cells using MACS cell separation kit as per the manufacturer's instructions, followed by flow cytometry. The CD34+ cells $\left(1 \times 10^{6}\right)$ were suspended in M199 culture medium (HyClone, Logan, UT, USA) and cultured in the same medium. The phenotype of the MSCs was identified with flow cytometry after labeling the cells $\left(1 \times 10^{6}\right)$ in $100 \mu 1$, with FITC-conjugated monoclonal antibodies for CD31, CD34 or rhodamine-conjugated CD133 antibody (1:100 dilution), with corresponding isotype controls. After incubation in the dark at $4^{\circ} \mathrm{C}$ for $30 \mathrm{~min}, 1 \mathrm{ml}$ of PBS+1\% FBS+0.1\% $\mathrm{NaN}_{3}$ was added and centrifuged at $800 \mathrm{x}$ g for $5 \mathrm{~min}$, and the supernatant was decanted. After washing twice in $3 \mathrm{ml}$ of PBS+1\% FBS $+0.1 \% \mathrm{NaN}_{3}$, the cells were processed for flow cytometry.

Immunofluorescent microscopy. Immunofluorescence phenotype analysis was performed on the third, fifth and seventh generation MSCs (the earliest primary continuous culture for 7 days). The cells were grown on gelatin-coated cover-slips in 6-well culture plates. At $60 \%$ confluency, the cells were washed with PBS 3 times, and fixed with acetone for $15 \mathrm{~min}$, followed by washing in PBS twice. Then the coverslips were left in $3 \% \mathrm{H}_{2} \mathrm{O}_{2}$ at room temperature for $15 \mathrm{~min}$, with agitation and washed with PBS twice. After blocking with sheep serum for $30 \mathrm{~min}$, and washing in PBS, primary antibodies (Ki67, anti-human factor VIII, anti-VEGF, CD31, CD34, CD133) were added (1:100 dilution) and incubated in humidified box at $37^{\circ} \mathrm{C}$ for $1 \mathrm{~h}$, followed by washing in PBS, twice. FITClabeled second antibody (1:200 dilution) was then added and incubated for another $1 \mathrm{~h}$, and then washed with PBS twice. Double labeling was carried out by incubating the coverslips with two antibodies. The coverslips were then observed under a fluorescent microscope (IX70, Olympus, Tokyo, Japan).

Growth and ultra structure of the MSCs. Cell proliferation was monitored with the 5th generation of cells by cell count every day. Growth and morphological characteristics of live cells were observed under inverted microscope. Transmission electron microscopic examination of human and canine EPCs was done under normal operation, and human EPCs were also examined by scanning electron microscopy (JEM-F200, Japan Electron Optics Laboratory Co., Ltd., Tokyo, Japan).

\section{Results}

Purification and cell culture of bone marrow-derived EPCs. Human (Fig. 1 A-D) and canine bone marrow stromal cells (Fig. 1E-H) showed similar changes towards EPCs during cell culture. After adhering to the dish surface for $24 \mathrm{~h}$, cell colonies formed, with the adherent cells extending 

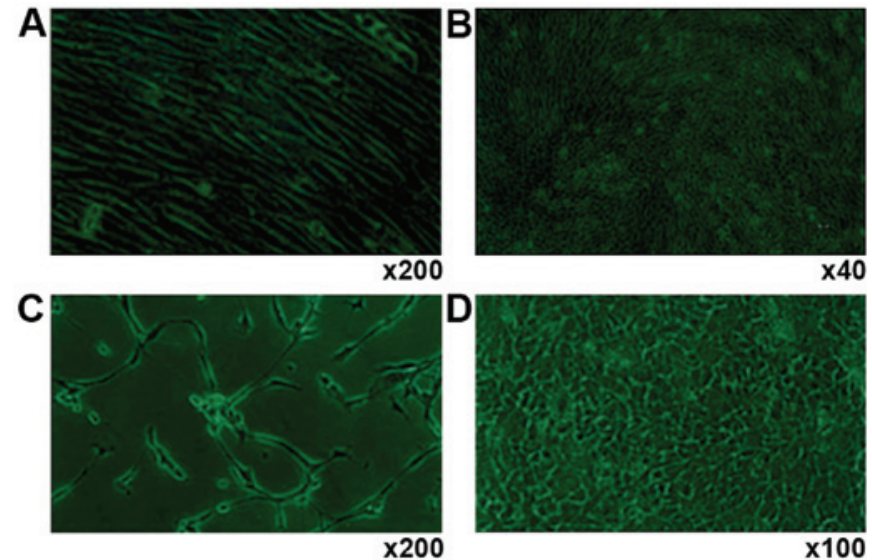

E
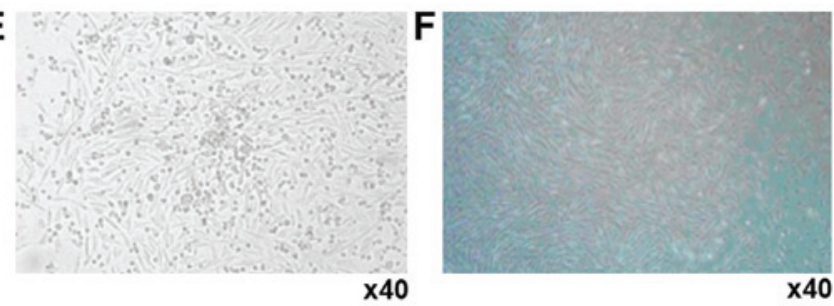

G

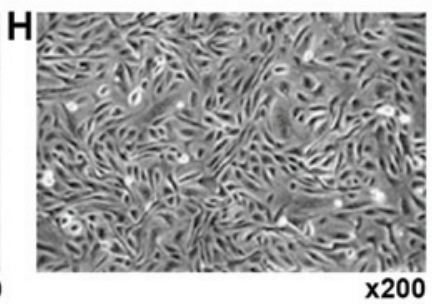

Figure 1. Formation of EPCs from human and canine bone marrow Magnification: (A) x200; (B) x40; (C) x200; (D) x100; (E) x40; (F) x40; (G) $\times 100$; (H) x200. EPCs, endothelial progenitor cells.

into spindle shape, and small cytoplasm and there was a small quantity of hemocytes (Fig. 1A and G). One week later, the spindle cells dominated in the culture dish, with large nucleus, and these cells grew in parallel arrangement and there were greatly reduced number of hemocytes (Fig. 1B and F). At this stage, the cells grew vigorously and could be passaged twice each week at a ratio of 1:3. The cells began to develop into short spindle shape (Fig. 1C and G). The cells also displayed a marked contact growth inhibition. After a month of continuous culture, cell morphology was stable even after 30 generations, and the cells were arranged in pebble shape and showed no aging-related phenomena or detachment (Fig. 1D and H).

Phenotype of the bone marrow-derived cells. We noted a gradual decline in the expression of CD31 in human- and canine-derived cells, during their multiplication. Thus, in the 5th generation CD31 expression was observed in $31.1 \%$ cells, which decreased to $18.6 \%$ in the 9 th generation and was completely absent by 15 th generation. On the other hand, the expression of factor VIII was strongly positive in the 15 th generation with $76 \%$ of cells showing a positive expression (Fig. 2). We did not observe any expression of Ki67 during the whole cell culture period.
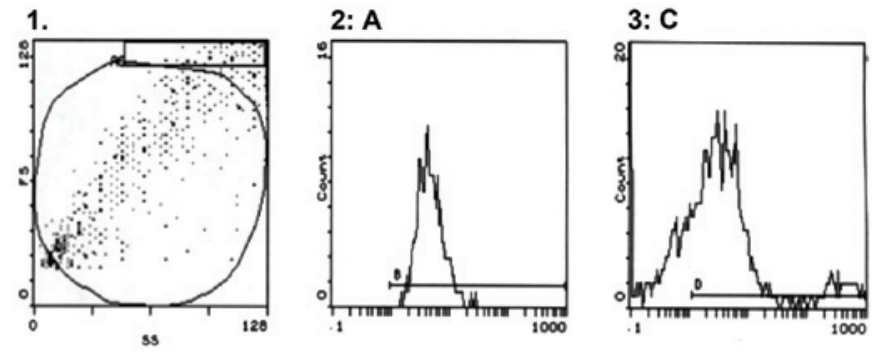

\begin{tabular}{|c|c|c|c|c|c|}
\hline Hist & Region ID & $\%$ & Count & Mn X & Mn Y \\
\hline 1 & C C & 38.9 & 4320 & 53.4 & 63.5 \\
\hline & A A & 11.3 & 1250 & 105.2 & 124.6 \\
\hline 2 & B B & 99.8 & 1247 & 5.31 & \\
\hline 3 & D D & 76.0 & 3283 & 7.79 & \\
\hline
\end{tabular}

Figure 2. Flow cytometry detection on factor VIII-FITC-labeled endothelial progenitor cells (EPCs).

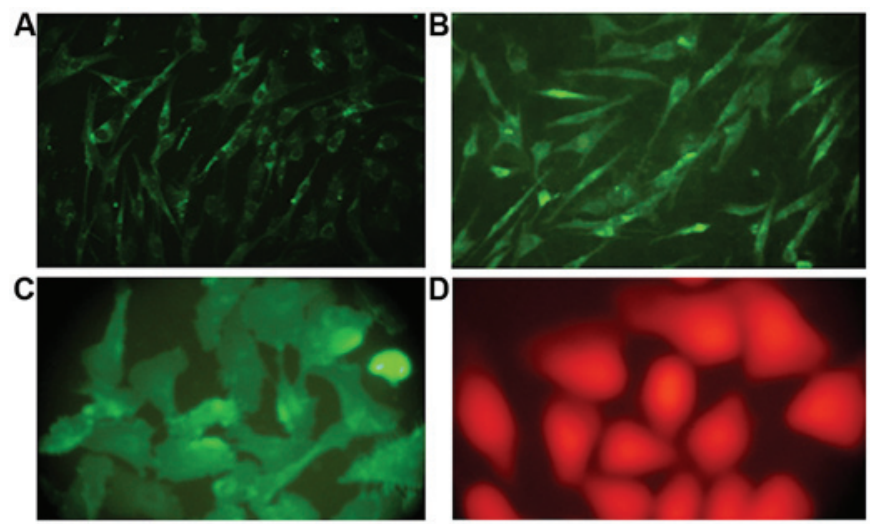

Figure 3. Immunofluorescence microscopic identification of the fifth generation cells. (A) Factor VIII-positive; (B) VEGF-positive; (C) CD133-positive; and (D) CD34-positive.

Immunofluorescence. Immunohistochemical examination of cells after staining with specific antibodies revealed that only a few mononuclear cells at the beginning of the cell culture expressed VEGF, while almost $35 \%$ of the cells showed positive factor VIII staining. As the culture progressed, the number of cells showing a positive expression of CD133 and VEGF gradually increased. By the 6th and 7th generation, factor VIII showed a strong positive expression in almost 99 and $95 \%$ of the cells from human and canine bone marrow, respectively (Fig. 3).

Growth curve and ultrastructure of the EPCs. EPCs from humans and canines were counted every day of the culture for five days, and from the growth curve, cell doubling time was calculated. This doubling time for human EPCs was $30.5 \mathrm{~h}$ (Fig. 4A; y=6.13+0.328x; correlation coefficient, $\mathrm{r}=0.977$ ) and for canine EPCs it was $13.4 \mathrm{~h}$ (Fig. 4B; $\mathrm{y}=1.196+0.06 \mathrm{x}$; correlation coefficient, $\mathrm{r}=0.978$ ).

Transmission electron microscopic examination of the ultrastructure of human-derived EPCs (Fig. 5A, C and E) and canine-derived EPC (Fig. 5B, D and F) revealed hyperchromatism on the periphery of cell nucleus, and that the cell nuclei 
A

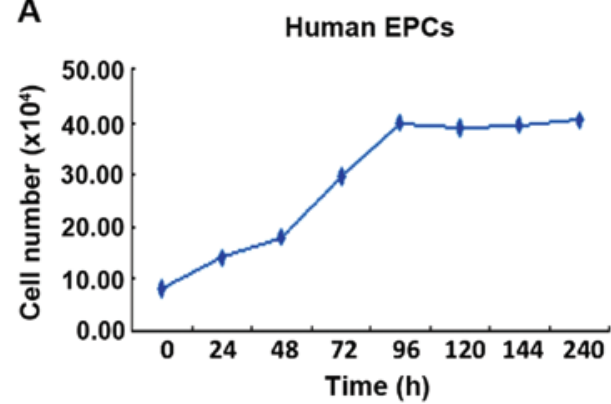

B

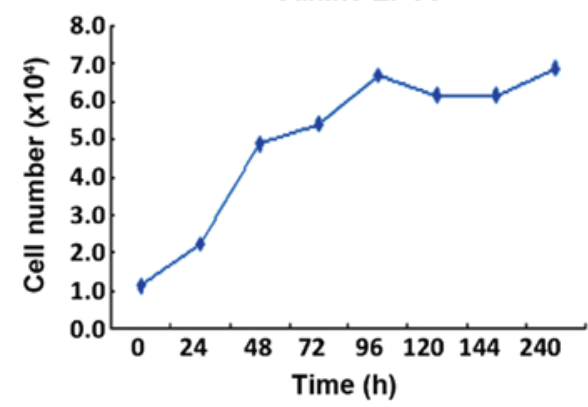

A
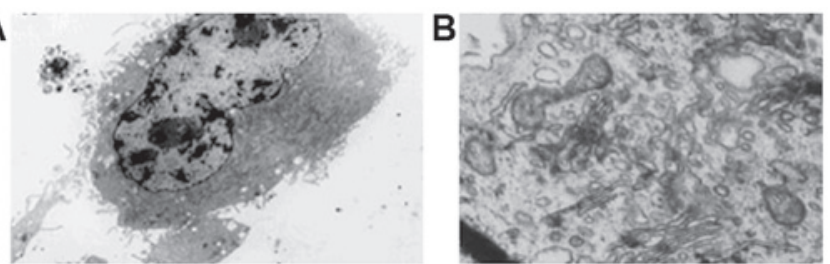

C
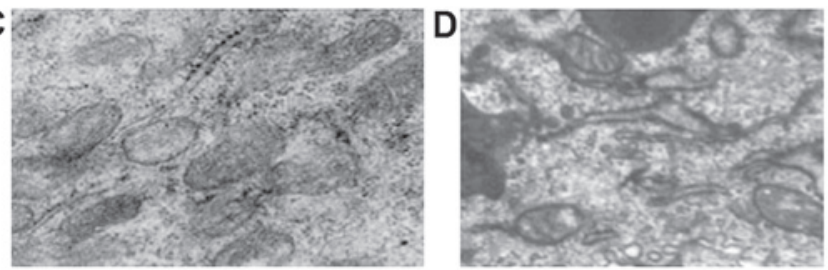

E
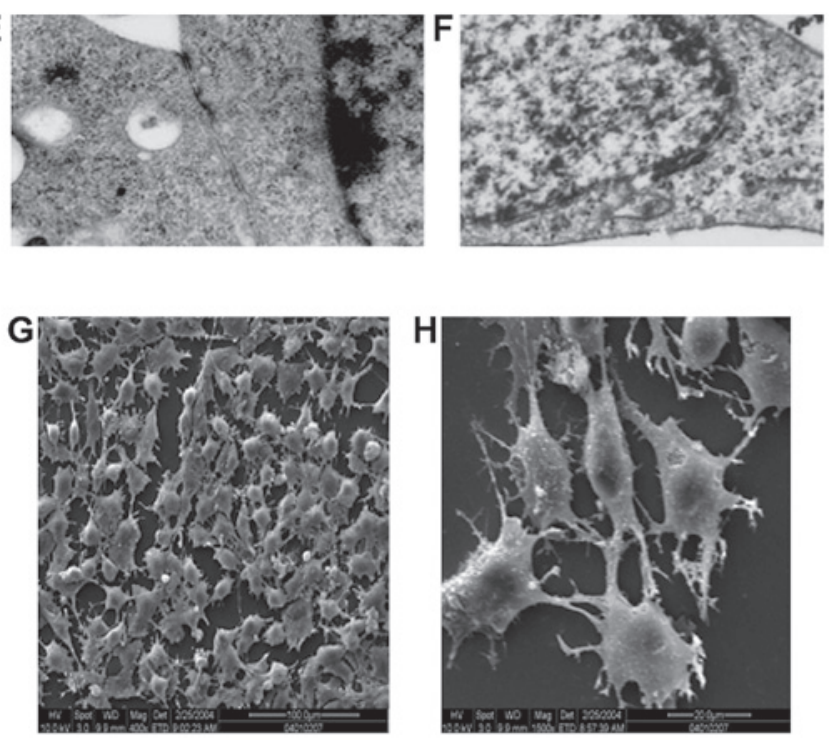

Figure 5. Electron microscopy of culture-induced bone marrow-derived cells. (A-F) Transmission electron microscopy (x30,000). (G and H) Scanning electron microscopy [magnification, $(\mathrm{G}) \mathrm{x} 400$ and $(\mathrm{H}) \mathrm{x} 1,500]$.

in human-derived cells (Fig. 5E). Gap junctions can strengthen the connection of the adjacent cells, but more importantly, these act as a communication structures of the cell, for information transfer between cells and are related to cell secretion, proliferation and differentiation. While in the canine-derived cells, Weibel-Palade bodies (Fig. 5B) with endothelial cell characteristics were observed in the endochylema, these bodies were encapsulated by monifilm structure and contained medium electron dense materials. Canaliculus separated by electron dense materials were identified within certain bodies. These different shapes probably represent different storage stages of factor von Willebrand.

Scanning electron microscopic observation revealed that the cells on the processed slides were found to be rounded or quasi-circular (Fig. 5G), with short and thick microvilli protruding on the cell surface, and cytoplasm extended and protruding towards the two poles (Fig. $5 \mathrm{H}$ ). This indicated that cells were quite active metabolically and functionally and had strong secretion capacity, and could absorb large quantity of nutrients to support growth and proliferation.

\section{Discussion}

Vascular development is a regulated process that consists of the proliferation and migration of adjacent vascular endothelial cells (angiogenesis), and the differentiation of EPCs into mesodermal precursor cells (vasculogenesis) (7-9). EPCs and HPC originate from the blood islands in the extraembryonic mesoderm in the embryonic stage (10). EPCs and HPCs have many common cell surface antigens, such as EGFR-2 (mouse Flk-1), Tie-2, and CD34 (11). Therefore, it is speculated that these cell types originate from the same precursor stem cell, the hematopoietic cells (12). In vitro, embryonic bodies differentiate into hematopoietic and vascular endothelial cells (13). After birth, EPCs, HSCs and MSC reside together in the bone marrow and are released from the bone marrow into the peripheral blood circulation (12).

Examination of cell surface markers and biological characteristics of vascular EPCs in the present study, revealed that EPCs are a subgroup of $\mathrm{CD} 34^{+}$cells. These EPCs after purification and subsequent cell culture with added VEGF and other growth factors may be induced into endothelial cells. Therefore, there is an absolute dependence on VEGFR-2 expression, which acted as the surface receptor for mediating the VEGF signals, in the early stage and during different stages of differentiation of vascular endothelial cells.

The findings of the present study support the hypothesis that a large scale preparation of EPCs that are capable of 
differentiating into vascular endothelial cells, can be prepared by continuous culture of cells from bone marrow-derived stromal cells. Such a supply of the EPCs can be of great value for various therapeutic applications.

\section{Acknowledgements}

The present study was supported by the National Natural Science Foundation of China (contract grant no. 81271304).

\section{References}

1. Spyridopoulos I and Andres V: Control of vascular smooth muscle and endothelial cell proliferation and its implication in cardiovascular disease. Front Biosci 3: d269-d287, 1998.

2. Ahn GO and Brown JM: Role of endothelial progenitors and other bone marrow-derived cells in the development of the tumor vasculature. Angiogenesis 12: 159-164, 2009.

3. Dimmeler S: Regulation of bone marrow-derived vascular progenitor cell mobilization and maintenance. Arterioscler Thromb Vasc Biol 30: 1088-1093, 2010.

4. Walter DH, Haendeler J, Reinhold J, Rochwalsky U, Seeger F, Honold J, Hoffmann J, Urbich C, Lehmann R, Arenzana-Seisdesdos F, et al: Impaired CXCR4 signaling contributes to the reduced neovascularization capacity of endothelial progenitor cells from patients with coronary artery disease. Circ Res 97: 1142-1151, 2005.
5. Rossi DJ, Jamieson CH and Weissman IL: Stem cells and the pathways to aging and cancer. Cell 132: 681-696, 2008.

6. Dimmeler S and Leri A: Aging and disease as modifiers of efficacy of cell therapy. Circ Res 102: 1319-1330, 2008.

7. Eguchi M, Masuda H and Asahara T: Endothelial progenitor cells for postnatal vasculogenesis. Clin Exp Nephrol 11: 18-25, 2007.

8. Asahara T, Masuda H, Takahashi T, Kalka C, Pastore C, Silver M, Kearne M, Magner M and Isner JM: Bone marrow origin of endothelial progenitor cells responsible for postnatal vasculogenesis in physiological and pathological neovascularization. Circ Res 85: 221-228, 1999.

9. Murasawa S and Asahara T: Endothelial progenitor cells for vasculogenesis. Physiology (Bethesda) 20: 36-42, 2005.

10. LaRue AC, Lansford R and Drake CJ: Circulating blood island-derived cells contribute to vasculogenesis in the embryo proper. Dev Biol 262: 162-172, 2003.

11. Case J, Mead LE, Bessler WK, Prater D, White HA, Saadatzadeh MR, Bhavsar JR, Yoder MC, Haneline LS and Ingram DA: Human CD $34^{+} \mathrm{AC} 133^{+} \mathrm{VEGFR}-2^{+}$cells are not endothelial progenitor cells but distinct, primitive hematopoietic progenitors. Exp Hematol 35: 1109-1118, 2007.

12. Khakoo AY and Finkel T: Endothelial progenitor cells. Annu Rev Med 56: 79-101, 2005.

13. Choi K, Kennedy M, Kazarov A, Papadimitriou JC and Keller G: A common precursor for hematopoietic and endothelial cells. Development 125: 725-732, 1998. 